\title{
DISTANCIAS MARITALES: SALTA (ARGENTINA)
}

\author{
MARITAL DISTANCES: SALTA (ARGENTINA)
}

\author{
Selene Pilar Torres ${ }^{1,2,3 *}$, María Virginia Albeza $2,3,4$ y Noemí Acreche $2,3,4$ \\ ${ }^{1}$ Consejo Nacional de Investigaciones Científicas y Técnicas (CONICET) \\ ${ }^{2}$ Consejo de Investigación Universidad Nacional de Salta (CIUNSa). Universidad Nacional de Salta. Salta. Argentina \\ ${ }^{3}$ Facultad de Humanidades. Universidad Nacional de Salta. Salta. Argentina \\ ${ }^{4}$ Facultad de Ciencias Naturales. Universidad Nacional de Salta. Salta. Argentina
}

\section{PALABRAS CLAVE Puna; Valle Calchaquí; Valle de Lerma; migración}

RESUMEN Se estimó la movilidad en once poblaciones de Puna, Valle Calchaquí y Valle de Lerma (Provincia de Salta) a partir de matrices de migración cónyuge-cónyuge, considerando las distancias entre los lugares de nacimiento de ambos. A partir de las distancias entre lugar de nacimiento y de residencia se estimó la migración diferencial por sexo en general, por zona geoestructural y por localidad. En general, los varones migran a mayores distancias que las mujeres. Para estimar patrilocalidad o matrilocalidad se consideraron las parejas con, al menos, un individuo local, tanto por zona como por localidad. En los Valles se observa un patrón de residencia matrilocal. Las tres zonas geoestructurales presentan una distribución asimétrica positiva, característica de poblaciones rurales endógamas. Del análisis de los resultados se desprende que, en las poblaciones estudiadas, el modelo de aislamiento por distancia no refleja la estructura marital de las mismas. Rev Arg Antrop Biol 21(2), 2019. doi: $10.24215 / 18536387 \mathrm{e} 005$

\section{KEY WORDS Puna, Calchaqui Valley, Lerma Valley, migration}

ABSTRACT Mobility was estimated in eleven populations of the Puna region, and the Calchaquí and Lerma Valleys (Salta) from marital migration matrices, considering the distances between birthplaces of the spouses. From distances between places of birth and of residence, differential migration by sex was estimated. In general, men migrate to greater distances than women. To estimate patri or matrilocality, couples with at least one local individual were considered, both by area and locality. A matrilocal residence pattern is observed in the valleys. The distribution of distances of the three regions showed a strong positive skewness, characteristic of inbred rural populations. In the studied populations, analysis of the results shows that the isolation-by-distance model does not reflect their marital structure. Rev Arg Antrop Biol 21(2), 2019. doi: $10.24215 / 18536387 \mathrm{e} 005$
Los procesos microevolutivos pueden derivar en una diferenciación genética entre poblaciones. Entre ellos, es necesario tener en cuenta la importancia que tiene el comportamiento migratorio de los individuos ya que las poblaciones no son sistemas cerrados y, por ende, pueden ocurrir intercambios genéticos entre ellas que pueden contribuir a reducir la divergencia.

Desde el punto de vista de la Genética de Poblaciones, la migración no sólo implica el movimiento de individuos sino también puede modificar la estructura genética de las poblaciones. Cuando la migración determina un flujo génico, no todos los migrantes, ya sea que entren o salgan de una población, son relevantes, sino sólo aquellos que dejan descendencia en la misma (migración efectiva: $\mathrm{m}_{\mathrm{e}}$ ) (Magalhaes y ArceGomez, 1987).

Si bien el flujo génico tiende a contrarrestar el efecto de la deriva y, en consecuencia, la diferenciación, se puede suponer que poblaciones separadas por cortas distancias geográficas son más semejantes entre sí en su estructura genética que poblaciones que se encuentren más distantes, ya que los cruzamientos al azar están limitados por la distancia (Wright, 1943). Así, los individuos tendrán mayor probabilidad de aparearse con vecinos que con aquellos que residen en localidades más alejadas, excepto durante

Financiamiento: CIUNSa Proyecto No2208.

*Correspondencia a: Selene Pilar Torres. Universidad Nacional de Salta. Avenida Bolivia 5150 (4400) Salta. Argentina. Email: selene_torres87@hotmail.com

Recibido 1 Junio 2018; aceptado 18 Marzo 2019

doi: $10.24215 / 18536387 \mathrm{e} 005$ 
expansiones demográficas o cuando las poblaciones se encuentran subdivididas por barreras geográficas y/o culturales (Barbujani, 2003).

Las estructuras demográfica y genética de las poblaciones se encuentran estrechamente relacionadas. En este sentido, la estructura demográfica refleja los procesos vinculados, entre otros, al nacimiento, muerte, migración y matrimonio.

Rothhammer (1977) señala que la estructura social de una población se encuentra estrechamente relacionada con la estructura matrimonial de la misma. En este sentido, uno de los aspectos más interesantes del análisis de esta estructura matrimonial está relacionado con la movilidad vinculada a la conformación de parejas, ya que los matrimonios proporcionan la posibilidad de examinar las interacciones bioculturales que afectan el mantenimiento de la variabilidad humana (Esparza Pages, 2004).

Cavalli-Sforza (1996) considera que, desde el punto de vista genético, los desplazamientos importantes son los cambios de residencia permanentes, especialmente aquellos que acompañan al nacimiento del individuo, matrimonio o nacimiento de su descendencia, ya que son estos movimientos los que pueden afectar la estructura genética de las poblaciones involucradas.

Por regla general, el matrimonio o conformación de pareja implica, en muchos casos, el desplazamiento de uno de los cónyuges, ya sea antes o después del mismo. Este movimiento puede realizarse dentro de la misma población o no, en cuyo caso supone un intercambio entre diferentes poblaciones. Roberts (1976) plantea que la movilidad asociada al matrimonio, puede ser entendida como la distancia existente entre los lugares de nacimiento de los cónyuges. Esta movilidad permite estimar el flujo génico entre una población y las localidades vecinas, aportando información acerca de si los matrimonios unen o no áreas genéticamente diferentes (Cavalli-Sforza y Bodmer, 1981; Sánchez Compadre, 1989).

La importancia de la determinación de esta movilidad y del flujo que implica, radica, como ya fuera señalado, en la posible modificación de la estructura genética de una población. La distribución de distancias maritales puede verse influenciada no sólo por la distancia geográfica en sí misma sino también por factores sociales, culturales, religiosos, entre otros, que tendrán correlación con la diferenciación genética entre poblaciones (Acreche, 2007). Una pareja, puede establecerse en una localidad cualquiera, pero es más probable que lo haga en la localidad de origen de alguno de los dos cónyuges.

Para estudiar las migraciones y las relaciones entre poblaciones se han propuesto varios modelos, entre los que se destacan el de Islas, el Stepping Stone y el de Aislamiento por Distancia.

El Modelo de Aislamiento por Distancia, desarrollado por Sewall Wright en 1943, es un modelo continuo en el que se supone que los individuos se aíslan por la distancia y representa aislados distribuidos continuamente en un espacio y conectados por niveles de migración proporcionales a sus distancias (Barbujani, 2003), es decir que la población no se encuentra subdividida en subpoblaciones donantes o receptoras de migrantes. En este modelo, los cruzamientos al azar están limitados por la distancia por lo que habrá una disminución del flujo génico a medida que aumente la distancia geográfica. Así, será más probable que los individuos de una población se apareen con individuos de localidades vecinas que con los de localidades más alejadas. Esto permite que los individuos puedan agruparse en "vecindarios" cuyos progenitores se pueden tratar como extraídos al azar. El tamaño del vecindario es función del desvío estándar de la distancia entre los lugares de nacimiento de los esposos o los padres y su descendencia, teniendo en cuenta, también, el número de individuos por unidad de distancia (Cavalli-Sforza y Bodmer, 1981).

El modelo de Aislamiento por Distancia se basa en la construcción de matrices, tanto de vinculación entre individuos (cónyuge-cónyuge; padres-descendientes), como de las distancias geográficas correspondientes.

Las matrices de migración describen los patrones de movilidad de una población y, en caso de migración marital, definen los patrones de búsqueda de pareja. En función de los acontecimientos vitales que se utilicen para construirla, se estarán considerando distintos aspectos de la movilidad de los individuos (Peña, 2001).

En este sentido, Bodmer y Cavalli-Sforza (1968) y Cavalli-Sforza y Bodmer (1981) plantean que la utilización de matrices de migración constituye la mejor técnica para predecir la deriva génica. Así, en función de qué es lo que vaya 
a estimarse, se obtendrán distintos tipos de distribuciones: a) si lo que se quiere visualizar es la distribución de las distancias entre los lugares de nacimiento de los progenitores y sus descendientes, se tendrán dos distribuciones: padredescendiente y madre-descendiente; b) si el objetivo es evaluar la endogamia, es conveniente estudiar la distribución de las distancias entre los lugares de nacimiento de los cónyuges. Para la distribución de las distancias entre los lugares de nacimiento y residencia de los maridos y las esposas la información puede obtenerse de certificados de matrimonio y/o de censos.

En este trabajo se obtuvieron las distancias entre los lugares de nacimiento esposo - esposa en localidades de la provincia de Salta (Argentina) con el objeto de poner a prueba la hipótesis del aislamiento por distancia y estimar la movilidad marital, patrilocalidad o matrilocalidad.

\section{MATERIAL Y MÉTODOS}

Los datos demográficos fueron obtenidos mediante censos domiciliarios realizados en once localidades de la provincia de Salta: cinco pertenecientes a la Puna, tres al Valle Calchaquí $y$ tres al Valle de Lerma. A partir de la información obtenida se elaboró una base de datos por localidad y zona y con el lugar de nacimiento se construyeron matrices cónyuge-cónyuge, disponiendo en las filas $(j)$ los lugares de nacimiento de las esposas y en las columnas (i) los de los esposos (Cavalli-Sforza y Bodmer, 1981), obteniéndose las distancias entre los lugares de nacimiento de los cónyuges y la migración diferencial por sexo.

La matriz cónyuge-cónyuge es una matriz rectangular con igual número de filas y columnas cuyo cuerpo está constituido por la frecuencia absoluta de las parejas conformadas por las diferentes combinaciones de lugares de nacimiento.

Para cada localidad y cada combinación de lugares de nacimiento en las diferentes matrices, se construyeron matrices triangulares de distancias geográficas entre los lugares de nacimiento de esposo-esposa. Esta matriz se caracteriza por tener ceros en la diagonal ya que ésta representa que ambos cónyuges nacieron en la misma localidad. Las distancias fueron medidas en kilómetros, en línea recta, entre pares de localidades, a través de la herramienta Google Earth y el mapa de la re- gión Norte del Automóvil Club Argentino (2015).

La decisión de medir las distancias en línea recta y no siguiendo las carreteras como plantean Cavalli-Sforza y Bodmer (1981), se debe a que, en la mayoría de las localidades que se analizan en el presente trabajo, los individuos se trasladan entre los distintos puestos en línea recta, siguiendo ya sea el camino más corto o el que presenta las menores dificultades topográficas, más que siguiendo las rutas convencionales, independientemente de la distancia que los separe.

También se obtuvieron matrices triangulares de distancias euclídeas entre los lugares de nacimiento, matrices con igual dimensión que las correspondientes a las de las distancias geográficas.

En los casos en los que el cónyuge no residiera en la localidad estudiada, pero se contara con el dato del lugar de nacimiento, la pareja se incluyó en la matriz. Por otro lado, para países o provincias en los que no se especifica una localidad, las distancias fueron medidas desde la capital de los mismos.

En este trabajo, además de la distancia en sí misma, las migraciones se definen en función de la zona geoestructural (Puna, Valle Calchaquí y Valle de Lerma). En este sentido, se consideraron como "Locales" a los individuos nacidos en la misma zona geoestructural y "Otro" a los nacidos fuera de ella.

Los patrones de búsqueda de pareja fueron evaluados a partir de las distancias existentes entre el lugar de residencia y de nacimiento para cada sexo. Para la estimación de la migración diferencial por sexo se consideró la totalidad de las parejas de las tres regiones y, por otro lado, todas aquellas parejas conformadas por, al menos, un individuo local, a nivel localidad y zona geoestructural, en función de la unidad de estudio trabajada. Para estimar patrilocalidad o matrilocalidad sólo se consideraron las parejas conformada por, al menos, un individuo local.

Se aplicó el test de Student para evaluar la migración diferencial por sexo y la prueba Chi cuadrado para patrilocalidad o matrilocalidad.

Para la puesta a prueba del Modelo de Aislamiento por Distancia, se analizaron las correspondientes matrices por localidad y la distribución de las distancias de los lugares de nacimiento de los cónyuges. A través del programa NTSYSpc versión 2.0 (Rohlf, 2000) se confrontaron las matrices de frecuencia (distancias 
euclídeas) y las de distancia geográfica a través del Test de Mantel, estimándose los correspondientes coeficientes de correlación $r$ y su nivel de significación $p$.

El tratamiento estadístico de los datos se realizó con el paquete SPSS for Windows, Versión 19.0.

\section{Poblaciones estudiadas}

En la región de la Puna se incluyeron las localidades de Chañarcito, Cobres, Olacapato, Santa Rosa de los Pastos Grandes y Tolar Grande, todas pertenecientes al Departamento de Los Andes, con excepción de Cobres (Departamento de La Poma); en el Valle Calchaquí, Cachi, San José y El Barrial, (Departamento de Cachi) y en el Valle de Lerma, La Isla, San Agustín (Departamento de Cerrillos) y Chicoana (zona urbana del Departamento homónimo) (Fig. 1).

Salta pertenece a la región del Noroeste Argentino (NOA) junto a Jujuy, Catamarca, Tucumán y Santiago del Estero. De acuerdo al Censo Nacional de Población, Hogares y Vivienda del año 2010 (INDEC, 2010), la región cuenta con 4.577.770 habitantes.

La provincia posee límites nacionales e internacionales; al Oeste limita con Chile, al Norte con Bolivia y Jujuy, al Este con Paraguay y con las pro- vincias de Formosa y Chaco y al Sur con las de Santiago del Estero, Tucumán y Catamarca. En ella se identifican diversas áreas diferenciadas por su clima, altitud, flora y fauna, relieve e hidrografía: Puna y Prepuna; Región de los Valles: Valle Calchaquí y Centrales y Región Chaqueña.

Esta diversidad ambiental determina diferencias en los recursos disponibles para las poblaciones humanas, lo que las ha llevado a presentar distintas estrategias al momento de su establecimiento y supervivencia.

Las poblaciones de la Puna se encuentran sometidas a condiciones de vida extremas debido a la baja presión de oxígeno y a las bajas temperaturas. Al ser una zona relativamente aislada, la base de las sociedades andinas es la unidad doméstica, orientándose su producción principalmente al autoabastecimiento complementado con la consecución de elementos para el consumo y con fuentes externas de trabajo.

La forma en que estas sociedades han resuelto la escasez estacional de recursos a la que las localidades puneñas se encuentran expuestas fue desarrollando un complejo adaptativo que incluye la transhumancia cíclica (Abeledo, 2014), subordinada a la disponibilidad de agua como condicionante para la presencia de pasturas (Fernández y Trillo, 2014) y participando del intercambio de productos

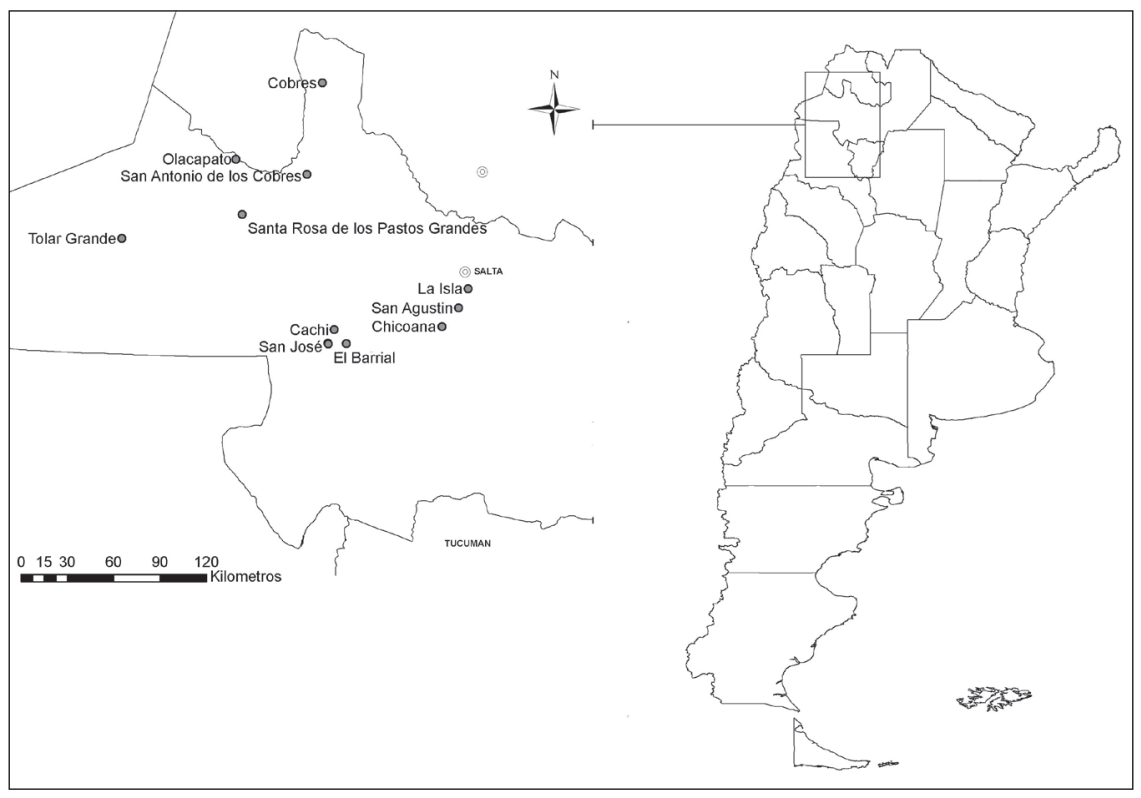

Fig. 1. Mapa de la provincia de Salta, Argentina. 
agrícolas con regiones aledañas. En cuanto a su patrón de asentamiento, está dado por la ausencia de sitios densamente poblados y la presencia de puestos de montaña, vinculados principalmente al pastoreo (Albeza, 1995; Caruso, 1995).

La región del Valle Calchaquí ocupa un sector de la Cordillera Oriental en el límite este de la Puna Argentina. En Salta, abarca los departamentos de Cachi, La Poma, San Carlos, Molinos y Cafayate. Su clima es cálido y seco, presentando una gran amplitud térmica. Las actividades humanas se encuentran condicionadas por las particularidades del clima y las escasas precipitaciones determinan que la actividad agrícola se realice únicamente bajo riego. Su población ha mantenido un crecimiento constante desde mediados del siglo XIX, aunque se encuentra desigualmente distribuida. Se practica la agricultura y la ganadería de subsistencia a la vez que se registran desplazamientos poblacionales, en épocas de cosecha, hacia valles más bajos.

El Valle de Lerma se encuentra en el centro de la provincia de Salta. Incluye los departamentos de La Caldera, Capital, Cerrillos, Chicoana, La Viña, Guachipas y Sudeste del de Rosario de Lerma. Su clima es subtropical serrano con una estación seca de abril a septiembre y las precipitaciones se concentran entre noviembre y marzo. La mayor parte de la superficie del suelo ha sido desmontada con el objeto de destinar las tierras al cultivo.

Desde mediados del siglo XX, la región experimentó un acelerado crecimiento, particularmente en la ciudad de Salta. A partir de 1940 se intensifica el cultivo de tabaco, convirtiéndose en una importante fuente de trabajo, aunque se caracteriza por la contratación de mano de obra no calificada, obreros golondrinas de otras localidades, provincias o países (Cámara del Tabaco de la Provincia de Salta, 1997).

\section{RESULTADOS}

Los datos analizados abarcan la totalidad de los individuos residentes en cada una de las localidades estudiadas y se registró, de ser posible, para los cónyuges no residentes, su lugar de nacimiento.

Se incluyeron un total de 2508 datos referidos a lugares de nacimientos de cónyuges: 400 de las localidades de la Puna, 1042 del Valle Calchaquí y 1066 del Valle de Lerma.

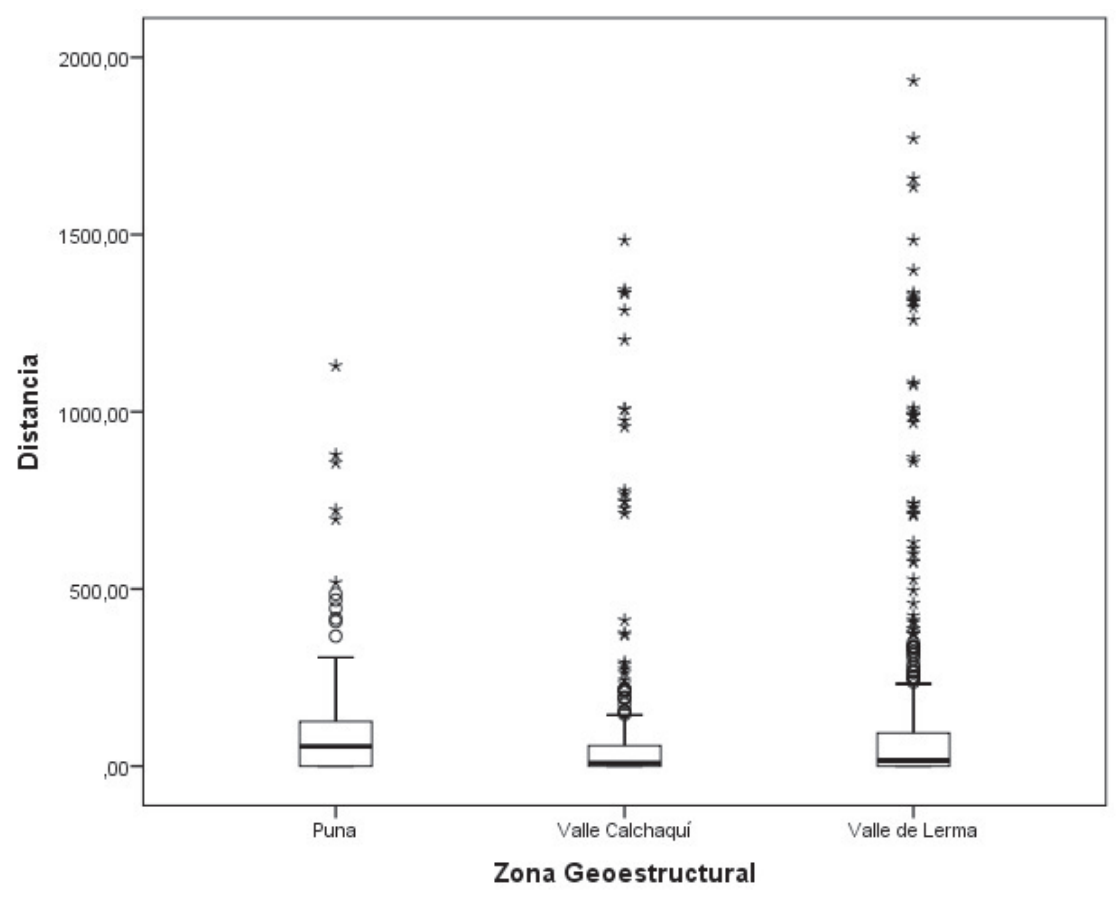

Fig. 2. Diagrama de cajas. 
En el boxplot (Fig. 2) se incluyeron sólo los individuos nacidos en países limítrofes con la provincia de Salta quedando excluidas siete parejas. La dispersión de los datos, al graficar la zona por la distancia, se observa que las muestras de la Puna y el Valle Calchaquí son menores que la del Valle de Lerma, región que presenta medias y varianzas mayores.

Pueden observarse una serie de "outliers", siendo el más extremo el de la pareja de Chicoana (Valle de Lerma) conformada por el esposo nacido en Formosa y la esposa en Temuco (Chile), con una distancia de 1934,31km. En la región del Valle Calchaquí, el caso más extremo $(1483,80 \mathrm{~km}$ de distancia) corresponde a la pareja de la localidad de Cachi, integrada por el esposo nacido en Buenos Aires y la esposa en Campamento Vespucio (Salta) y en la Puna, la mayor distancia $(1129,85 \mathrm{~km})$ es la de la pareja con esposo nacido en Chile y esposa en el paraje Puesto Sey (Jujuy).

Para el total de las parejas, la distancia mínima es cero y corresponde a los dos cónyuges nacidos en el mismo lugar, la distancia promedio es de $134,55 \mathrm{~km}(\mathrm{~S}=615,04 \mathrm{~km})$ y la máxima observada $(10636,59 \mathrm{~km})$ es la de una pareja de la localidad de Chicoana (Valle de Lerma) conformada por el esposo de Francia y la esposa de Cachi (Valle Calchaquí).

En cuanto a la distancia marital, los matrimonios se agruparon en las siguientes categorías: $0-99 \mathrm{~km} ; 100-199 \mathrm{~km} ; 200-299 \mathrm{~km} ; 300$ - 399km; 400 - 499km; 500 - 599km; más de $600 \mathrm{~km}$. Aquí también puede observarse la gran dispersión de las distancias del Valle de Lerma (Fig. 3). Asimismo, es posible visualizar en las tres zonas que la clase $0-99 \mathrm{~km}$ muestra la frecuencia más elevada con una distribución asimétrica positiva.

Se aplicó el test de Student para comparar las distancias medias de las tres zonas, siendo en todos los casos las diferencias significativas: Valle Calchaquí - Valle de Lerma $(\mathrm{p}<0,001)$; Puna - Valle de Lerma, $(p=0,007)$ y Puna - Valle Calchaquí $(\mathrm{p}=0,040)$.

En el Análisis de la Varianza de un factor (zona) las diferencias significativas entre distancias maritales medias se observan entre el Valle Calchaquí $(68,32 \mathrm{~km})$ y el Valle de Lerma $(211,89 \mathrm{~km})$.

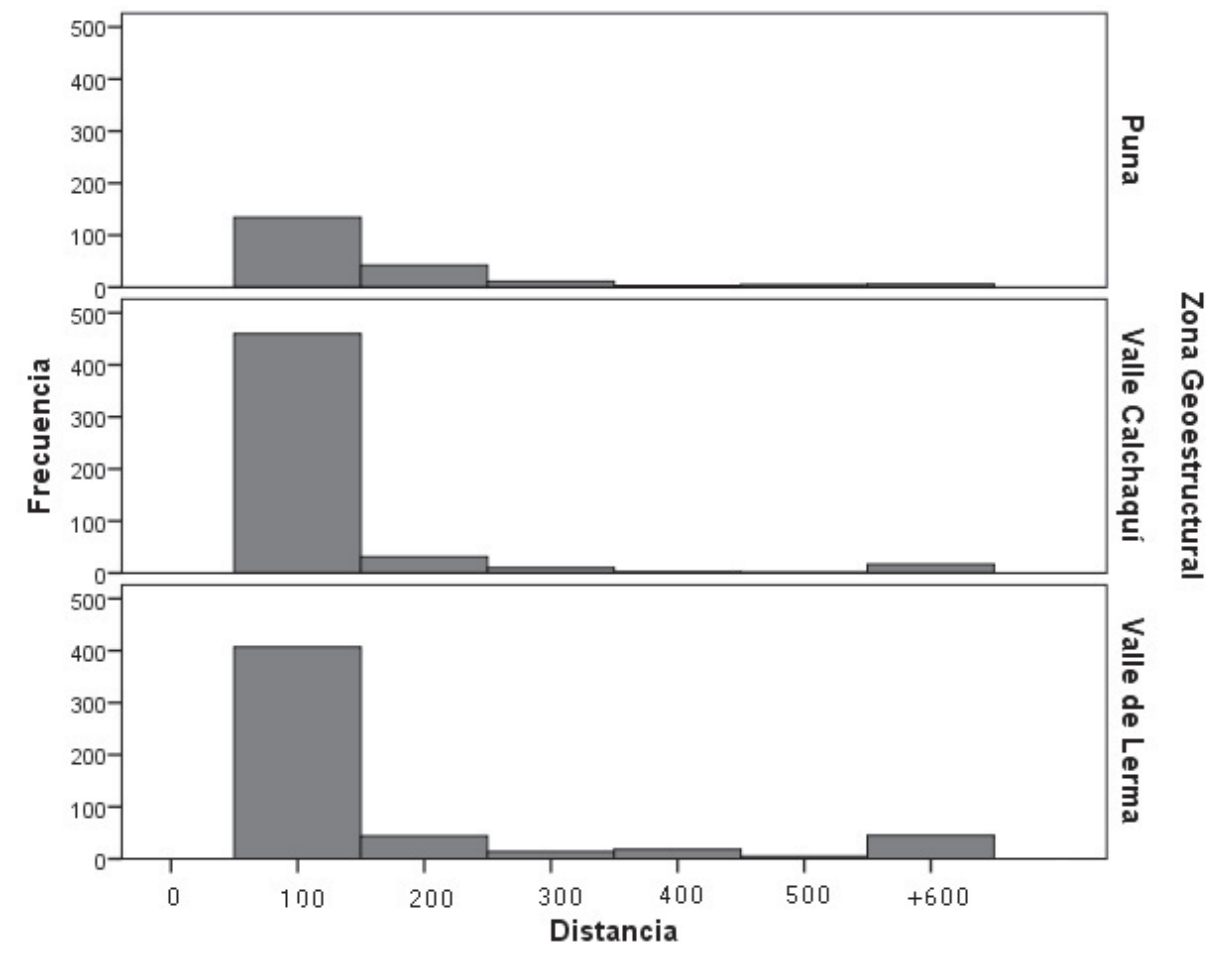

Fig. 3. Histograma de distancias por zona. 
Del análisis de la distribución de las medias de las distancias maritales por localidad (Fig. 4), se observa que las localidades de la Puna presentan valores medios superiores a los de las del Valle Calchaquí y del Valle de Lerma, con excepción de la media de Chicoana, cuyo valor es superior al del resto de las localidades.

En cuanto a la migración diferencial por sexo, considerando la totalidad de las parejas, existen diferencias significativas entre las distancias medias entre varones y mujeres, siendo los esposos los que migran más lejos que las esposas $(117,88 \mathrm{~km}$ y $63,79 \mathrm{~km}$, respectivamente, $p=0,005)$. Cuando el análisis se realiza por zona geoestructural, en la Puna son las mujeres las que migran a mayores distancias, no siendo significativas las diferencias. Tanto en el Valle Calchaquí como en el Valle de Lerma la situación es inversa, aunque sólo se observan diferencias significativas entre las medias de varones $(180,48 \mathrm{~km})$ y mujeres $(69,38 \mathrm{~km})$ del Valle de Lerma $(p=0,006)$.

En cuanto a la migración diferencial por sexo por localidades, en siete de las once anali- zadas, las mujeres migran a mayores distancias (Tabla 1). En el caso de las localidades puneñas, excepto Chañarcito, las esposas migran a mayores distancias. En las localidades del Valle Calchaquí, en Cachi son los varones los que migran a mayores distancias, mientras que en San José y El Barrial son las mujeres, pero las diferencias no son significativas en ninguno de estos casos. En Chicoana (Valle de Lerma) se registra la única diferencia significativa $(\mathrm{p}=0,006)$, siendo los varones los que migran a mayores distancias. Las siete parejas mencionadas anteriormente son de esta localidad, y en seis de ellas, son los varones los nacidos en países no limítrofes (Perú, Guatemala, Francia, España y Portugal).

$\mathrm{Al}$ analizar la migración diferencial por zona geoestructural, considerando las parejas conformadas por, al menos, un individuo "Local" (nacido en la zona geoestrucutural o localidad, respectivamente) y "Otro" (nacido fuera de la región o localidad), la diferencia es significativa en el Valle Calchaquí $(\mathrm{p}=0,028)$ siendo las distancias mayores en los varones que en las mujeres.

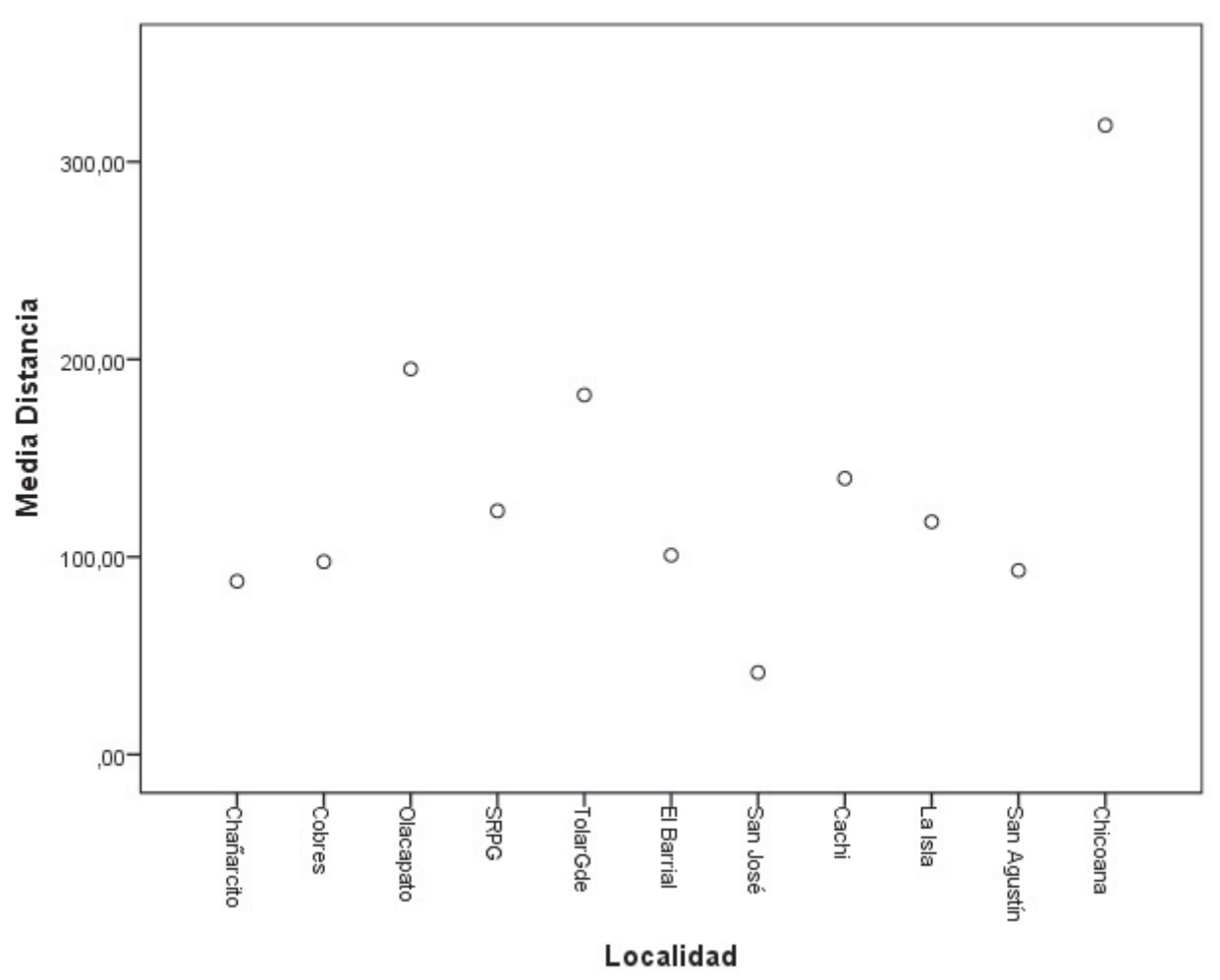

Fig. 4. Distancias promedio por localidad. 
TABLA 1. Distancias ponderadas por zona, localidad y sexo considerando la totalidad de las parejas

\begin{tabular}{|c|c|c|c|}
\hline Zona & Localidad & Sexo & $\begin{array}{c}\text { Distancia } \\
\text { ponderada }(\mathrm{km})\end{array}$ \\
\hline \multirow{10}{*}{ Puna } & \multirow{2}{*}{ Chañarcito } & $\mathrm{m}$ & 94.71 \\
\hline & & $\mathrm{f}$ & 51.85 \\
\hline & \multirow{2}{*}{ Cobres } & $\mathrm{m}$ & 47.85 \\
\hline & & $\mathrm{f}$ & 80.93 \\
\hline & \multirow{2}{*}{ Olacapato } & $\mathrm{m}$ & 43.07 \\
\hline & & $\mathrm{f}$ & 83.62 \\
\hline & \multirow{2}{*}{ Santa Rosa } & $\mathrm{m}$ & 21.52 \\
\hline & & $\mathrm{f}$ & 54.42 \\
\hline & \multirow{2}{*}{ Tolar Grande } & $\mathrm{m}$ & 146.88 \\
\hline & & $\mathrm{f}$ & 249.92 \\
\hline \multirow{6}{*}{$\begin{array}{c}\text { Valle } \\
\text { Calchaquí }\end{array}$} & \multirow{2}{*}{ Cachi } & $\mathrm{m}$ & 66.43 \\
\hline & & $\mathrm{f}$ & 52.71 \\
\hline & \multirow{2}{*}{ El Barrial } & $\mathrm{m}$ & 7.49 \\
\hline & & $\mathrm{f}$ & 30.25 \\
\hline & \multirow{2}{*}{ San José } & $\mathrm{m}$ & 13.10 \\
\hline & & $\mathrm{f}$ & 28.15 \\
\hline \multirow{6}{*}{$\begin{array}{l}\text { Valle de } \\
\text { Lerma }\end{array}$} & \multirow{2}{*}{ La Isla } & $\mathrm{m}$ & 90.76 \\
\hline & & $\mathrm{f}$ & 63.26 \\
\hline & \multirow{2}{*}{ San Agustín } & $\mathrm{m}$ & 60.17 \\
\hline & & $\mathrm{f}$ & 88.42 \\
\hline & \multirow{2}{*}{ Chicoana } & $\mathrm{m}$ & 193.12 \\
\hline & & f & 68.64 \\
\hline
\end{tabular}

m:masculino; f:femenino.

Cuando este análisis se realiza por localidad, son los hombres los que migran a mayores distancias, aunque sólo Cachi registra valores significativos entre distancias medias $(\mathrm{p}=0,001)$ (Tabla 2$)$.

Con el objetivo de estimar patrilocalidad o matrilocalidad tanto por zona geoestructural como por localidad, se consideraron las parejas con un cónyuge "Local". En cuanto al análisis por zona, la tabla de contingencia muestra resultados significativos para el Valle Calchaquí y el Valle de Lerma, con las esposas como mayor componente Local ( $p<0,001$ en ambos casos).

A nivel de localidades de la Puna, Chañarcito y Santa Rosa de los Pastos Grandes presentan matrilocalidad, mientras que en Tolar Grande y Cobres los esposos son locales, con
TABLA 2. Distancias ponderadas por zona, localidad y sexo con al menos un "Local"

\begin{tabular}{cccc}
\hline Zona & Localidad & Sexo & $\begin{array}{c}\text { Distancia } \\
\text { ponderada }(\mathrm{km})\end{array}$ \\
\hline & Chañarcito & $\mathrm{m}$ & 126.40 \\
& & $\mathrm{f}$ & 20.63 \\
& Cobres & $\mathrm{m}$ & 28.27 \\
Puna & Olacapato & $\mathrm{f}$ & 43.62 \\
& & $\mathrm{f}$ & 215.47 \\
& Santa Rosa & $\mathrm{m}$ & 75.99 \\
& & $\mathrm{f}$ & 72.87 \\
& Tolar Grande & $\mathrm{m}$ & 306.69 \\
& & $\mathrm{f}$ & 140.88 \\
& Cachi & $\mathrm{m}$ & 34.07 \\
Valle & & $\mathrm{f}$ & 7.69 \\
Ealchaquí & El Barrial & $\mathrm{m}$ & 19.15 \\
& & $\mathrm{f}$ & 5.63 \\
& San José & $\mathrm{m}$ & 4.00 \\
& & $\mathrm{f}$ & 26.62 \\
& & $\mathrm{~m}$ & 7.71 \\
& La Isla & $\mathrm{f}$ & 4.42 \\
& & $\mathrm{~m}$ & 0.00 \\
& San Agustín & $\mathrm{f}$ & 40.59 \\
& & $\mathrm{~m}$ & 61.64 \\
& Chicoana & $\mathrm{f}$ & 71.54 \\
\hline
\end{tabular}

m:masculino; f:femenino.

resultados significativos en esta última localidad $(\mathrm{p}=0,023)$. En Olacapato, se observa la misma cantidad de esposos que de esposas locales.

En cuanto a las localidades del Valle Calchaquí, las esposas son locales en Cachi y El Barrial con valor significativo sólo para Cachi $(p<0,001)$, contrariamente a lo observado en San José, que presenta patrilocalidad $(\mathrm{p}=0,039)$. Entre las localidades del Valle de Lerma, las esposas son locales en La Isla y Chicoana, con valores significativos para esta última $(\mathrm{p}<0,001)$. En San Agustín se detecta patrilocalidad con la particularidad que en este análisis no se observan mujeres locales.

Se realizó el test de Mantel entre matrices para cada localidad, considerando las matrices cónyuge - cónyuge, para el esposo y la esposa 
por separado con las respectivas matrices de distancias geográficas de los lugares de nacimiento. Se estimaron los coeficientes $r$ y sus respectivos niveles de significación $p$. En el caso de que la distancia entre lugares de nacimiento determine las uniones conyugales, se espera correlación significativa negativa entre matrices. Como puede observarse en la Tabla 3, todas las correlaciones computadas, excepto cuatro, son negativas. Sin embargo, sólo una es significativa: las esposas de Tolar Grande, por lo que debería rechazarse el Modelo de Aislamiento por Distancia.

\section{DISCUSIÓN}

Los resultados obtenidos en el presente trabajo indicarían que el aislamiento y la estructuración poblacional no estarían dados por la distancia geográfica en sí misma sino por la pertenencia a una región natural con características particulares como las que aquí se analizan, que abarcan aspectos no sólo naturales sino también sociales y culturales. Por ejemplo, las poblaciones pertenecientes a la región de la Puna, se encuentran a distancias muy grandes, superiores en muchos casos a las distancias que separan las localidades puneñas de las de otras zonas geográficas. Esto se encuentra en concordancia con lo planteado por Acreche y Albeza (2010a, b) a partir del análisis de datos demográficos y genéticos, que señalan que las

TABLA 3. Correlación entre distancias maritales y distancias geográficas

\begin{tabular}{cccc}
\hline Zona & Localidad & Esposo & Esposa \\
\hline & Chañarcito & -0.276 & -0.093 \\
& Cobres & -0.036 & -0.164 \\
Puna & Olacapato & -0.283 & -0.223 \\
& Santa Rosa & -0.180 & -0.168 \\
& Tolar Grande & 0.106 & -0.297 \\
Valle & San José & -0.141 & -0.164 \\
Calchaquí & El Barrial & -0.212 & -0.093 \\
& Cachi & 0.149 & 0.001 \\
Valle de & La Isla & -0.201 & -0.196 \\
Lerma & San Agustín & -0.035 & -0.232 \\
& Chicoana & -0.006 & 0.010 \\
\hline
\end{tabular}

poblaciones se encuentran aisladas reproductivamente y que la Homogamia es propia de las unidades geoestructurales antes que de las poblaciones locales.

Todas las áreas geográficas muestran una distribución asimétrica positiva de las distancias entre los lugares de nacimiento de los cónyuges que, de acuerdo a lo señalado por Swedlund (1972), sería característica de los apareamientos humanos. En este sentido, es típica de poblaciones rurales endógamas (Cavalli-Sforza y Bodmer, 1981; Sánchez Compadre, 1989) y de aquellas que no suelen utilizar medios de transporte modernos (Cavalli-Sforza y Bodmer, 1981). Esto se ve reflejado particularmente entre las localidades de la Puna y del Valle Calchaquí, cuyos habitantes suelen trasladarse caminando entre los puestos de la región, independientemente de las distancias que los separan.

A su vez, el hecho de que la mayor cantidad de matrimonios se observe en la clase $0-99 \mathrm{~km}$, coincide con lo planteado por Jeffries, Harrison, Hiorns y Gibson (1976) en cuanto a los menores valores de distancia al matrimonio entre grupos agrícolas, aunque estos autores lo relacionan con individuos relativamente no móviles en el ámbito rural. En el análisis de movimientos pre y post-marital, los autores plantean que éstos suelen ser menores en los grupos agrícolas, con matrimonios en su mayoría locales, que entre individuos cuyas ocupaciones pueden ser gerenciales o profesionales, entre otras.

En el análisis de distancias ponderadas por sexo, considerando la totalidad de los datos, se observó que son los varones los que migran a mayores distancias con diferencias significativas, situación que se reproduce también en el Valle de Lerma y en Chicoana (en el análisis por zona geoestructural y por localidad, respectivamente). Esta diferencia significativa podría obedecer al tamaño de la población analizada y a su vez podría estar determinando las diferencias no sólo en el Valle de Lerma sino también al ser considerados varones y mujeres sin especificar localidad o zona. Esto estaría en correspondencia con lo planteado en 1978 por Fuster y colaboradores (Sánchez Compadre, 1989) respecto a que los valores medios en las distancias al matrimonio, se ven afectados por aquellos matrimonios cuyos cónyuges proceden de lugares muy distantes, como se observa en las parejas de 
Chicoana conformadas por individuos de países no limítrofes con la provincia de Salta.

En el Valle Calchaquí, tomando las distancias con, al menos, un cónyuge Local, la diferencia es significativa. En este caso, podría ser Cachi la localidad que esté determinando esta diferencia en las medias entre esposos y esposas.

Si bien se estima que el $70 \%$ de las sociedades humanas son patrilocales (Hamilton, Stoneking y Excoffier, 2005; Oota, Settheetham-Ishida, Tiwawech, Ishida y Stoneking, 2001; Seielstad, Minch y Cavalli-Sforza, 1998), dos de las tres zonas geoestructurales estudiadas (Valle Calchaquí y Valle de Lerma) y la mayor cantidad de localidades analizadas presentan matrilocalidad. En el caso de la Puna, si bien hay mayor cantidad de varones locales, la diferencia no es significativa, lo que podría estar relacionado con lo que sucede en la localidad de Olacapato que, como ya se mencionó, tiene la misma cantidad de esposos y esposas locales. A su vez, es posible que la falta de significación de los valores esté vinculada a la poca cantidad de parejas residentes con al menos un componente.

Es probable que el patrón de residencia matrilocal reflejado por la mayoría de las poblaciones, se encuentre relacionado con la movilidad de las mismas, particularmente de los varones, no sólo como una respuesta adaptativa a condiciones ambientales particulares de cada región, sino también como respuesta al acceso a pasturas, mano de obra en centros mineros, como sucede en las localidades de la Puna; o traslado hacia valles más bajos en épocas de cosecha, como se observa en las localidades del Valle de Lerma, como Chicoana, donde se cultiva tabaco, una importante fuente de trabajo pero que, como ya fue mencionado, se trata de obreros golondrinas que vienen de otras localidades o regiones.

En función de los datos aquí analizados, el Modelo de Aislamiento por Distancia no puede ser acreditado. Como se planteara anteriormente, se esperaría correlación positiva si la distancia entre los lugares de nacimiento determinara las uniones conyugales, lo que no sucede. Por otra parte, si la distribución de las distancias fuera aleatoria, se esperaría un número aproximadamente semejante de correlaciones positivas y negativas, lo cual tampoco ocurre.

\section{CONCLUSIONES}

A partir de los resultados obtenidos de la correlación entre distancias maritales y sus correspondientes distancias geográficas, se concluye que, aunque el modelo de aislamiento por distancia no refleja la estructura marital de las localidades estudiadas, no debe ser descartado completamente.

En cuanto a la constitución de parejas, las tres zonas geoestructurales analizadas presentan una distribución leptocúrtica, característica de poblaciones rurales endógamas, lo que podría estar significando que la búsqueda del cónyuge se da al interior de cada región. Esto reflejaría que el aislamiento y la estructuración de la población estaría dado por la pertenencia a una región natural con características particulares más que a la distancia geográfica en sí misma. En Chicoana, Valle de Lerma (zona geoestructural) y considerando a todos los individuos censados, los varones migran a mayores distancias que las mujeres, con diferencias significativas entre las medias de ambos sexos. Se observa matrilocalidad en los Valles, no así en la región de la Puna.

\section{LITERATURA CITADA}

Abeledo, S. (2014). Pastoreo transhumante a comienzos de un nuevo siglo: su vigencia en Santa Rosa de los Pastos Grandes (Departamento de Los Andes, Salta). Andes, 28.

ACA - Automovil Club Argentino. (2015). Hoja de Zona 1. Región Norte. Argentina.

Acreche, N. (2007). Aislamiento reproductivo y migración. Sesión de Trabajo $\mathrm{N}^{\circ} 1$ : Movimientos étnicos migratorios en el NOA. SEPOSAL 2005. Seminario Internacional de Población y Sociedad En América Latina. 1, 183-189.

Acreche, N., y Albeza, M. V. (2010a). El siglo de las migraciones: entre el pluralismo y la fusión. Primer simposio internacional interdisciplinario Aduanas del Conocimiento La traducción y la constitución de las disciplinas entre el Centenario y el Bicentenario. Consultado en: http://www.expoesia.com/media/ponencia_acreche $\% 20 y \% 20$ Albeza.pdf

Acreche, N., y Albeza, M. V. (2010b). Selección de parejas y homogamia en Salta. Revista Argentina de Antropologia Biologica, 12, 71-78.

Albeza, M. V. (1995). Deriva Génica: estructura poblacional en poblaciones andinas. Tesis Profesional. Facultad de Ciencias Naturales, Universidad Nacional de Salta, Salta.

Barbujani, G. (2003). Migration and drift.Encyclopedia of Life Sciences, 1-5. doi:/doi.org/10.1002/9780470015902. a0005450.pub2

Bodmer, W. F., y Cavalli-Sforza, L. L. (1968). A migration matrix model for the study of random genetic drift. $\mathrm{Ge}$ netics, 59(4), 565-592. 
Cámara del Tabaco de la Provincia de Salta. (1997). Informe de Producción. Recuperado de https://www.ctsalta. com.ar/

Caruso, G. (1995). Deriva génica y polimorfismos hematológicos en Santa Rosa de los Pastos Grandes (Región de la Puna - Salta). Tesis de Grado. Facultad de Ciencias Naturales, Universidad Nacional de Salta.

Esparza Pages, M. (2004). Biodemografia Del Delta De L'Ebre: Estructura Matrimonial. Tesis Doctoral. Universitat de Barcelona.

Cavalli-Sforza, L. L. (1996). Genes, Pueblos y Lenguas. Barcelona, España: Crítica.

Cavalli-Sforza, L. L., y Bodmer, W. F. (1981). Genética de las Poblaciones Humanas.. Barcelona, España: Omega,.

Cavalli-Sforza, L. L., y Bodmer, W. F. (1981). Genética de las Poblaciones Humanas. Barcelona, España: Omega.

Esparza Pages, M. (2004). Biodemografia del Delta de L'Ebre: estructura matrimonial. Tesis Doctoral, Departament de Biología Animal. Universitat de Barcelona. Recuperado en http://diposit.ub.edu/dspace/handle/2445/35884

Fernández, D., y Trillo, J. (2014). Transhumancia y territorio en las yungas de Jujuy . En III Jornadas Nacionales sobre estudios regionales y mercados de trabajo. Universidad Nacional de Jujuy (Facultad de Cs. Económicas y Unidad de Investigación en Comunicación, Cultura y Sociedad de la Facultad de Humanidades y Cs. Sociales) y Red SIMEL, San Salvador de Jujuy (pp. $0-13)$.

Hamilton, G., Stoneking, M., y Excoffier, L. (2005). Molecular analysis reveals tighter social regulation of immigration in patrilocal populations than in matrilocal populations. Proceedings of the National Academy of Sciences, 102(21), 7476-7480. doi.org/10.1073/ pnas. 0409253102

INDEC. (2010). Censo Nacional de Población, Hogares y Vivienda. Ministerio de Economía y Finanzas Públicas. Recuperado de http://www.censo2010.indec.gov.ar/
Jeffries, D. J., Harrison, G. A., Hiorns, R. W., y Gibson, J. B. (1976). A note on marital distances and movement, and age at marriage, in a group of oxfordshire villages. Journal of Biosocial Science, 8(2), 155-160. doi. org/10.1017/S0021932000010592

Magalhaes, J. C. M., y Arce-Gomez, B. (1987). Study on a Brazilian Isolate. I. Population Structure and Random Genetic Drift. Human Heredity, 37, 278-284.

Oota, H., Settheetham-Ishida, W., Tiwawech, D., Ishida, T., y Stoneking, M. (2001). Human mtDNA and Y-chromosome variation is correlated with matrilocal versus patrilocal residence. Nature Genetics, 29(1), 20-21. Recuperado en https://doi.org/10.1038/ng711

Peña, J. A. (2001). Estructura genética de la población del Valle de Orozco (País Vasco , España ) a partir de diferentes matrices de migración, (1970). Antropo 1-10. (www.didac.ehu.es/antropo).

Roberts, D. (1976). Les concepts d'isolats. En L'étude des isolats (pp. 75-92). París, Ed. INED.

Rohlf, F. J. (2000). NTSYSpc: numerical taxonomy and multivariate analysis system Version 2.0. Applied Biostatistic, New York, N.Y, 1998.

Rothhammer, F. (1977). Genetica de poblaciones humanas. Programa Regional de Desarrollo Científico y Tecnológico. Departamento de Asuntos Científicos. Secretaría General de la OEA. Serie de Biología. Monografía $\mathrm{N}^{\circ} 15$.

Sánchez Compadre, E. (1989). Babia: biodemografia y estructura familiar. Madrid, Universidad de León.

Seielstad, M. T., Minch, E., y Cavalli-Sforza, L. L. (1998). Genetic evidence for a high female migration rate in humans. Nature Genetics, 20(3), 278-280.

Swedlund,A. C. (1972). Observations on the concept of neighbourhood knowledge and the distribution of marriage distances. Annals of Human Genetics, 35, 327330.

Wright, S. (1943). Isolation by distance. Genetics, 28(2), 114-138. 